\title{
Revealing the morphology and the magnetic properties of single buried cobalt-ZnTPP hybrid interfaces by FNR spectroscopy
}

\author{
Garen Avedissian ${ }^{1}$, Jacek Arabski $^{1}$, Jennifer A. Wytko ${ }^{2}$, Jean Weiss ${ }^{2}$ and Christian Meny ${ }^{{ }^{*}}$ \\ ${ }^{1}$ Institut de Physique et Chimie des Matériaux de Strasbourg, Université de Strasbourg, CNRS \\ UMR 7504, 23 rue de Loess, BP 43, F-67034 Strasbourg Cedex 2, France \\ ${ }^{2}$ Institut de Chimie de Strasbourg, Université de Strasbourg, CNRS UMR 7177, 4 rue Blaise \\ Pascal, F-67081 Strasbourg Cedex, France \\ *e-mail : Christian.Meny@ipcms.unistra.fr
}

\begin{abstract}
The deeply buried, yet most important part of any spintronic device is the interface. This is even more interesting and much more complex when soft, light materials like organic molecules are in contact with an inorganic metallic electrode. Hence, exceptional care is required to better understand the phenomena driven by this type of organic/inorganic interfaces. To this end, ferromagnetic nuclear resonance (FNR) spectroscopy studies were performed to investigate the morphology and the magnetic propuries of the hybrid organic-inorganic interfaces when zinc tetra-phenyl porphyrin ( $\mathrm{Z} \cdot \mathrm{CP}$ molecules are at the vicinity of ferromagnetic metallic cobalt (Co) layers. The ETh experimental results show that when ZnTPP is deposited on top of Co the resulting inieface is smoother and sharper compared to the more extended interface obtained when C is eposited on top of ZnTPP. The shape of the spectra suggests that no chemical bonds ta k. place between the interfacial Co atoms and the ZnTPP molecules and that interactions it thic interfaces are governed by weak van der Waals forces. Finally, FNR also showed that tiie magnetic anisotropy at the Co-ZnTPP hybrid interfaces is reduced compared to the nas netic anisotropy of the Co atoms inside the Co films.
\end{abstract}

\section{Introduction}

Carbon-based organic compounds are expected to enhance the performances and functionalities of spintronic devices because of the large spin coherence length and long spin relaxation time expected in molecules. Many interesting physical properties have been observed in metal/organic hybrid systems [1-5]. The interfaces play a big role in these effects and it was even suggested that the properties of devices could be tuned by controlling the morphology of the interfaces. This resulted in the proposed concept of "spinterface" science [6]. However, before controlling and tuning interface morphologies and their physical properties, it is required to correlate existing interface morphologies to the sample properties. The morphology of the interface will depend on many parameters like roughness, atomic diffusion, chemical bonds etc. In this context, zinc tetra-phenyl porphyrin (ZnTPP) molecules have been chosen to build hybrid organic/inorganic heterostructures to study the morphological properties of the hybrid interfaces buried within the heterostructures. Because of the organic nature of one of the layers, conventional characterization techniques are often difficult to use especially when post-growth sample processing is required. In this work a non-invasive 
ferromagnetic nuclear resonance (FNR) [7,8] spectroscopic method has been developed to investigate the structure and the morphology of buried Co-ZnTPP hybrid interfaces.

FNR corresponds to nuclear magnetic resonance (NMR) when it is applied to study ferromagnetic systems. It is also sometimes called zero field NMR or internal field NMR. NMR is widely used in biology or chemistry, but its use to study ferromagnetic nanostructures is much scarcer. Yet, it has been shown that FNR is a very efficient tool to study the structure and morphology of interfaces in thin films, multilayers [9-17] and even devices [18]. FNR also provides unique insight into the understanding of the physicochemical properties of assemblies of nano-objects [19-23].

In a previous work, FNR has already been used to probe the continuity of the ZnTPP molecular layers embedded between ferromagnetic electrodes [24]. It was shown that to fully separate the ferromagnetic electrodes a minimum deposited thickness of 15 monolayers (ML) of ZnTPP is required. Making use of this knowledge, the morphology of the Co-ZnTPP hybrid interfaces has been investigated by building a series of ZnTPP/Co(t)/ZnTPP hybrid sandwiches. As already demonstrated for inorganic films, studying a series of samples that are dependent on the Co layer thickness allows determining the extension of the interfaces $[7,8,18]$. Surprisingly, this method revealed very extended Co-ZnTPP interfers with unexpected FNR contributions. To understand this phenomenon, an original samp's architecture has been implemented to investigate individually the buried bottom ZnTFP, $\mathrm{C}$ interface and the buried top Co/ZnTPP interface. It showed that when ZnTPP is deposied $\mathrm{c}$. top of Co, the interface is quite sharp whereas the interface is much wider and invo rec a much larger amount of Co atoms when Co is deposited on top of the ZnTPP layer. Althoris asymmetric interfaces have already been evidenced in multilayers through FNR s.ecr. modelling [15], it is the first time that single buried interfaces have been directly investigated by FNR. These investigations also showed that no specific and well-defined en ronment could be attributed to the interfaces formed by the Co atoms and the ZnTPP organic layer. This suggests that there are no strong chemical bonds between the surface Co atoms and the ZnTPP molecules and that the interactions at the interfaces are governed by weak van der Waals (vdW) forces. Finally, since the local magnetic anisotropy can also be probed by FNR [7,8], it is shown that the Co atoms at the interfaces experience a smaller magnetic anisotropy field than the Co atoms in the centre of the Co films.

\section{Materials and methods}

Two sets of heterostructures were prepared. First, the Co layers sandwiched by two ZnTPP films:

$\mathrm{Si} / \mathrm{SiO}_{2}(500 \mathrm{~nm}) / \mathrm{Cu}(25 \mathrm{~nm}) / \mathrm{Fe}(30 \mathrm{~nm}) / \mathrm{ZnTPP}(20 \mathrm{ML}) / \mathrm{Co}(t$ in $n m) / \mathrm{ZnTPP}(20 \mathrm{ML}) / \mathrm{Fe}(30$ $\mathrm{nm}) / \mathrm{Cr}(100 \mathrm{~nm})$, with Co thickness ranging from $2 \mathrm{~nm}$ to $6 \mathrm{~nm}$

Second, the samples designed for studying the single buried interfaces:

$\mathrm{Si} / \mathrm{SiO}_{2}(500 \mathrm{~nm}) / \mathrm{Cu}(25 \mathrm{~nm}) / \mathrm{Fe}(30 \mathrm{~nm}) / \mathrm{Co}(6 \mathrm{~nm}) / \mathrm{ZnTPP}(20 \mathrm{ML}) / \mathrm{Cr}(100 \mathrm{~nm})$

and the reversed architecture: 
The copper $(\mathrm{Cu})$ seed layers, the chromium $(\mathrm{Cr})$ capping layers as well as the iron $(\mathrm{Fe})$ layers were deposited by direct current $(\mathrm{dc})$ sputtering and under an argon pressure of $4.5 \times 10^{-4} \mathrm{mbar}$ in a standard sputtering chamber at a rate of $0.6 \AA / \mathrm{sec}, 0.5 \AA / \mathrm{sec}$ and $0.25 \AA / \mathrm{sec}$, respectively. The organic films are deposited in an organic molecular beam epitaxy chamber (OMBE). A total of 20 monolayers (ML) of analytically pure, in-lab synthesized, ZnTPP molecules were sublimed under $2 \times 10^{-9} \mathrm{mbar}$ of deposition pressure and at a rate of 0.055 monolayer $/ \mathrm{sec}$ (ML/sec with $1 \mathrm{ML}=3.5 \AA$; monitored with quartz). The Co films were deposited in an ultrahigh vacuum (UHV) chamber by thermal evaporation, with a flux of $0.16 \AA / \mathrm{sec}$ under a deposition pressure of $2 \times 10^{-9}$ mbar. All deposition chambers are connected under vacuum therefore the samples can be transferred from one chamber to another without breaking the UHV conditions.

In order to check that the Co layers are not contaminated during the deposition of the ZnTPP films two test Co films were prepared. One of the test samples was capped directly after the deposition of the Co film while the second one was introduced in the organic chamber. After ZnTPP started to sublimate, the test Co film was kept in the organic chamber during the same time as the regular samples but the ZnTPP crucible shitiar was kept closed. After removal from the organic chamber the sample was capped and its natieization curve compared the one of the Co film directly capped after the Co deposition without being introduced into the organic chamber. The magnetization of the two sampl 6 where very similar and did not show any difference in saturation magnetization. It slows inat the sublimation of ZnTPP does not deteriorate the chamber base pressure and dos rot result in the contamination of the Co film surface.

All FNR measurements were perforred in a homemade state of the art FNR set up. Regular NMR spin echo method has been used (r.lse length: $3.2 \mu \mathrm{s}$, delay: $3 \mu \mathrm{s})$. The integrated spinecho intensity was recorded using a broadband pulsed spectrometer with phase-sensitive detection and automated frequency scanning. In FNR, like in all NMR techniques, the degeneracy of the nuclear spins energy levels is lifted by a static magnetic field. The main particularity of FNR is that the static field already exists inside the ferromagnetic samples and has for origin the spontaneous magnetization of the sample. The main contribution to this internal static field is the so called the hyperfine field. Therefore, determining the nuclei resonance conditions is performed by frequency sweeping. The second particularity of FNR is that the amplitude of the radiofrequency (RF) field (the frequency swept field) experienced by the nuclei is enhanced by the magnetic susceptibility of the samples. Therefore, the optimum RF field power required to obtain the maximum FNR signal depends on the magnetic properties of the samples and has to be determined for each sample. The detailed experimental procedure is explained in $[7,8]$. This procedure allows to get FNR amplitudes that represent the true distribution of the probed atoms (nuclei) versus the RF field frequency. In addition, this experimental procedure also allows measuring the local magnetic stiffness (proportional to the reciprocal of the local susceptibility) in the sample [7,8]. This is represented by a frequency dependent restoring field. This restoring field characterizes the torque experienced by the local magnetic moments when their direction is slightly tilted from their position at rest. Depending 
on the nature of the samples it can be identified as a local effective anisotropy field, or exchange field, or pinning field... In the samples under investigation in this work the restoring field can be identified as the in plane local magnetic anisotropy field of the samples. The FNR measurements were performed at $1.8 \mathrm{~K}$ and the samples' surface area was of the order of 3 $\mathrm{cm}^{2}$. All the spectra are recorded from $50 \mathrm{MHz}$ to $320 \mathrm{MHz}$ with $0.6 \mathrm{MHz}$ steps. They are measured typically during 12 hours' accumulation time resulting in the averaging of about $10^{6}$ spectral measurements. As the thickness of the samples is much thinner than the RF penetration length, several micrometers for the considered frequency range, the full stack FNR signal contribution is retrieved. With these experimental conditions the set-up sensitivity is better than 0.1 atomic layer of $\mathrm{Co}$ (or $0.02 \mathrm{~nm}$ of $\mathrm{Co}$ ). The only post measurement normalization that is performed is to divide the recorded FNR intensity by the samples' surface area so that any discrepancy in the size of the samples is removed and their respective FNR intensities can be compared directly.

\section{Results and discussion}

For all samples, the ZnTPP films were chosen to be 20 monolayers thick $(\approx 7 \mathrm{~nm})$ to ensure the continuity of the molecular layers [24]. It has been shown that this thickness value prevents the migration of metallic atoms through the organic film [ $: 4$. . However, to ascertain the continuity of the organic films, additional $30 \mathrm{~nm}$-thick Fe layer vere added at the top and bottom of the architecture. As shown in [24] (and by the $\mathrm{Cu} / \mathrm{Co} / \mathrm{He}$ sample spectrum in black dashed line in figure 1) the contact of Fe with Co would resu'i in ingh frequency contributions in the FNR spectra $[25,26]$ and would directly reveal any iisitpion in the organic films.

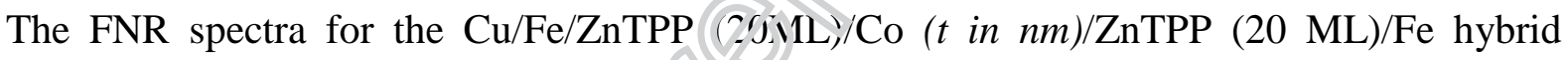
sandwich heterostructures are given in F zure 1. No FNR signal was observed above $250 \mathrm{MHz}$, which confirms that the ZnTPP layer: are trick enough to form continuous molecular films. It also shows that no Co-ZnTPP intertarial contribution is found at high frequency. Indeed, chemical bonds between Co and organics can give rise to high frequency contributions as it has been shown in the case of Co atoms bonded to carbon nanotubes [27]. Using the method established for the study of inorganic films and multilayers, the FNR spectra are normalized to the total surface area of the samples $[8,18]$. With this normalization process, the contributions in the spectra resulting from the $\mathrm{ZnTPP} / \mathrm{Co} / \mathrm{ZnTPP}$ interfaces should show no evolution in their spectral shape once the interfaces are completed. Only the contribution of the bulk part (Co situated far from the interfaces) will increase with the increasing thickness of the Co film. Figure 1 shows that as the Co thickness increases, the position of the main line becomes better defined and reaches a position of $224 \mathrm{MHz}$ close to that expected for Co atoms in a hexagonal closed pack (hcp) crystal structure having its magnetization axis perpendicular to the c-axis of the hcp crystal cell [25] ([0001] growth direction of the Co film with in plane easy magnetisation direction).

Whatever the morphology of the samples (bulk [25,26], films [7,8] or nanoparticles [19]) the main line corresponding to pure Co surrounded by other Co atoms will give no contributions outside the frequencies ranging from $200 \mathrm{MHz}$ to $250 \mathrm{MHz}$. As mentioned above no contribution has been observed above $250 \mathrm{MHz}$, therefore only the FNR intensity at low 
frequency ( $<200 \mathrm{MHz}$ ) has to be attributed to the Co/ZnTPP and ZnTPP/Co interface contributions. Whereas below about $120 \mathrm{MHz}$ all spectra are superimposed (within experimental errors), between $120 \mathrm{MHz}$ and $200 \mathrm{MHz}$ the spectra intensities continue to increase surprisingly until a Co thickness of $5 \mathrm{~nm}$ (the 5 and $6 \mathrm{~nm}$ low frequency spectra are superimposed, within experimental error bars). This shows a complex interface behaviour and suggests extended interface regions.

When interface regions are extended, it is not unusual for the interfaces to involve some fraction of Co atoms that are no longer ferromagnetic. These nonmagnetic Co atoms will not be detected by FNR. To probe the presence of non-ferromagnetic Co atoms, the total intensity of the FNR spectra as a function of the deposited Co thickness is represented in Figure 2. As expected, since the spectra intensities are normalized to the samples' surface area, the FNR integral intensity increases linearly with the increase of the deposited Co thickness (Figure 2, black symbol). However, the linear fit (Figure 2, black line) clearly shows that the total FNR intensity does not pass through the origin when extrapolated to zero Co thickness. From the intercept of the linear fit, the amount of Co that is no longer ferromagnetic can be determined. About 1.4 $\mathrm{nm}$ of Co are not ferromagnetic at $1.8 \mathrm{~K}$ (temperature of measurements) and hence not detected in the FNR spectra.

These dead layers can have several origins. The firs ne can be a partial oxidation of the Co films. As described in the methods section, it ins been checked that the sublimation of the ZnTPP film does not result in the contaminatior of the Co film surface. The efficiency of the capping layer has also been checked by track ng the magnetic properties of the samples over several months. No ingress of oxygen could he c videnced at this time scale. It has also been reported than when Co is deposited on S ( 2 t t $)$ e first layers can get oxidized [28], however in our samples the Co films are separater r, $; 25 \mathrm{~nm}$ thick $\mathrm{Cu}$ seed layers. Therefore, oxidation of the Co film can most likely be rul ou. Dead layers can also result from interfacial strains [29] however it looks unlikely that eprta ial strains can be induced on the Co films by the mechanically soft organic films. Dead layers can also result from amorphous phases but as the Co films are deposited at room temperature amorphous phases can only result from alloying with an alien element [10]. Nor Co-Fe nor Co-Zn alloys can produce amorphous phases. Therefore, most likely the observed dead layers result from Co atoms that did diffuse deep into the ZnTPP molecular films when forming the two hybrid interfaces. This has already been suggested in the study of the growth of the ZnTPP films [24]. 


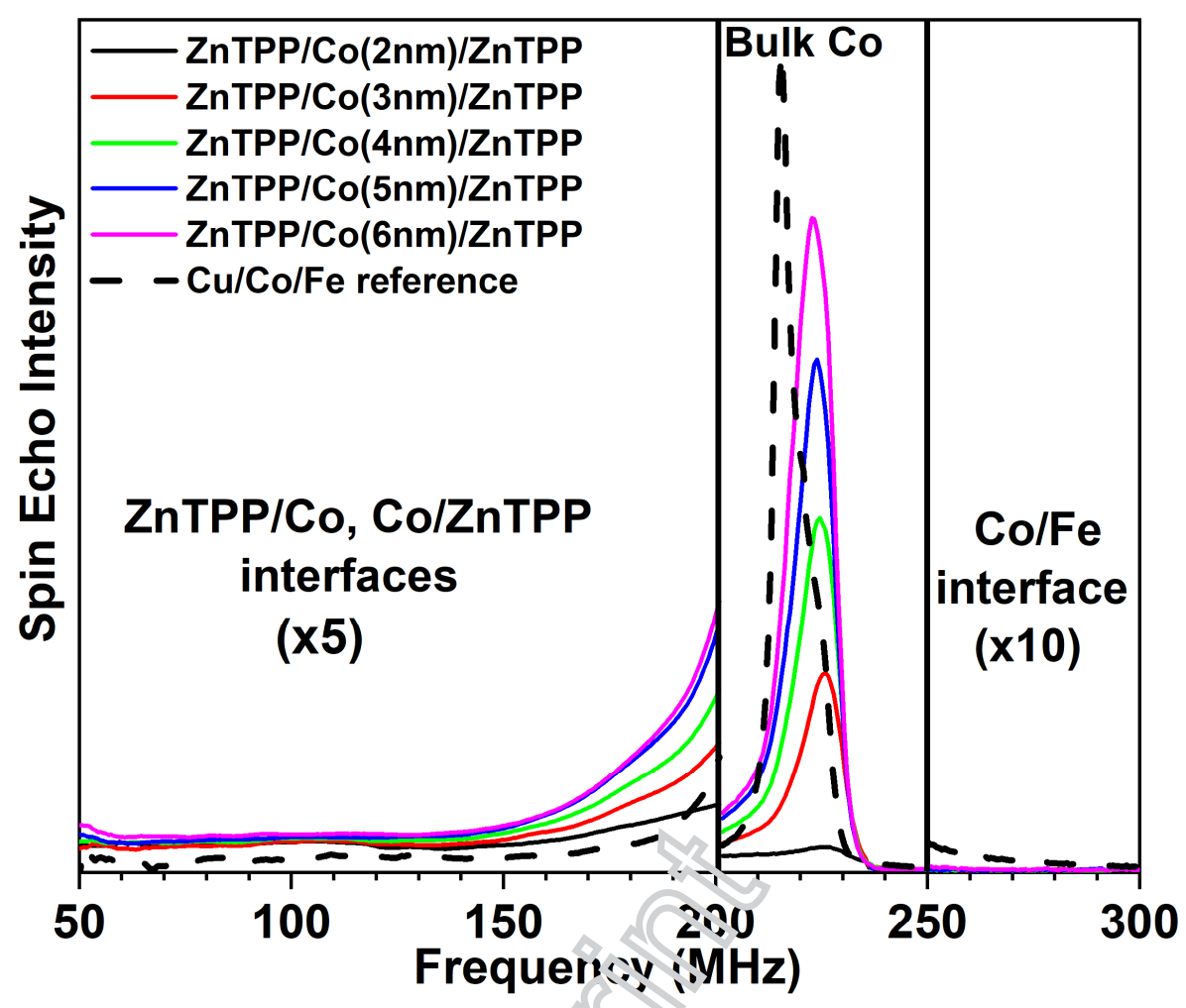

FIG. 1: Full lines: FNR spectra of the C to $6 \mathrm{~nm}$. The signal between $200 \mathrm{MHz}$ an $\cong 50 \mathrm{MHz}$ corresponds to the Co atoms situated far from the interfaces and that are sirmonded by other Co atoms only. The main line is at $224 \mathrm{MHz}$ close to the one expected rer Julk hcp Co. Between $120 \mathrm{MHz}$ and $200 \mathrm{MHz}$ the FNR intensity increases with the increase in Co thickness up to a deposited thickness of 5 nm. Below $200 \mathrm{MHz}$ the 5 and $6 \mathrm{~nm}$ spectra overlap. Black dashed line: Spectrum of a $\mathrm{Cu} / \mathrm{Co} / \mathrm{Fe}$ reference sample showing at low frequency the $\mathrm{Cu} / \mathrm{Co}$ interface and at high frequency the $\mathrm{Co} / \mathrm{Fe}$ interface [24]. The main line position is $217 \mathrm{MHz}$ since the Co film grows with an fcc structure on top of $\mathrm{Cu}$.

The plot of the spectra integral intensities for frequencies below $200 \mathrm{MHz}$ (interface range) is also shown in Figure 2. Since the spectra are normalized to the surface area of the samples, these integral intensities should reach a constant value once the interfaces are completed. This is obviously not the case in these samples. Only the integral intensities for the 5 and $6 \mathrm{~nm}$ samples become similar. This can be clearly seen from Figure 1 where the 5 and $6 \mathrm{~nm}$ low frequency spectra are superimposed (within the FNR experimental error bars). Since the contributions below $200 \mathrm{MHz}$ are out of the Co bulk frequency range it shows that the interfaces continue to build up until $5 \mathrm{~nm}$ of Co thickness. However, it is rather surprising that this "building up of interfaces" results in contributions above $120 \mathrm{MHz}$ only. One explanation might be that the top and bottom Co-ZnTPP interfaces are highly asymmetrical. 


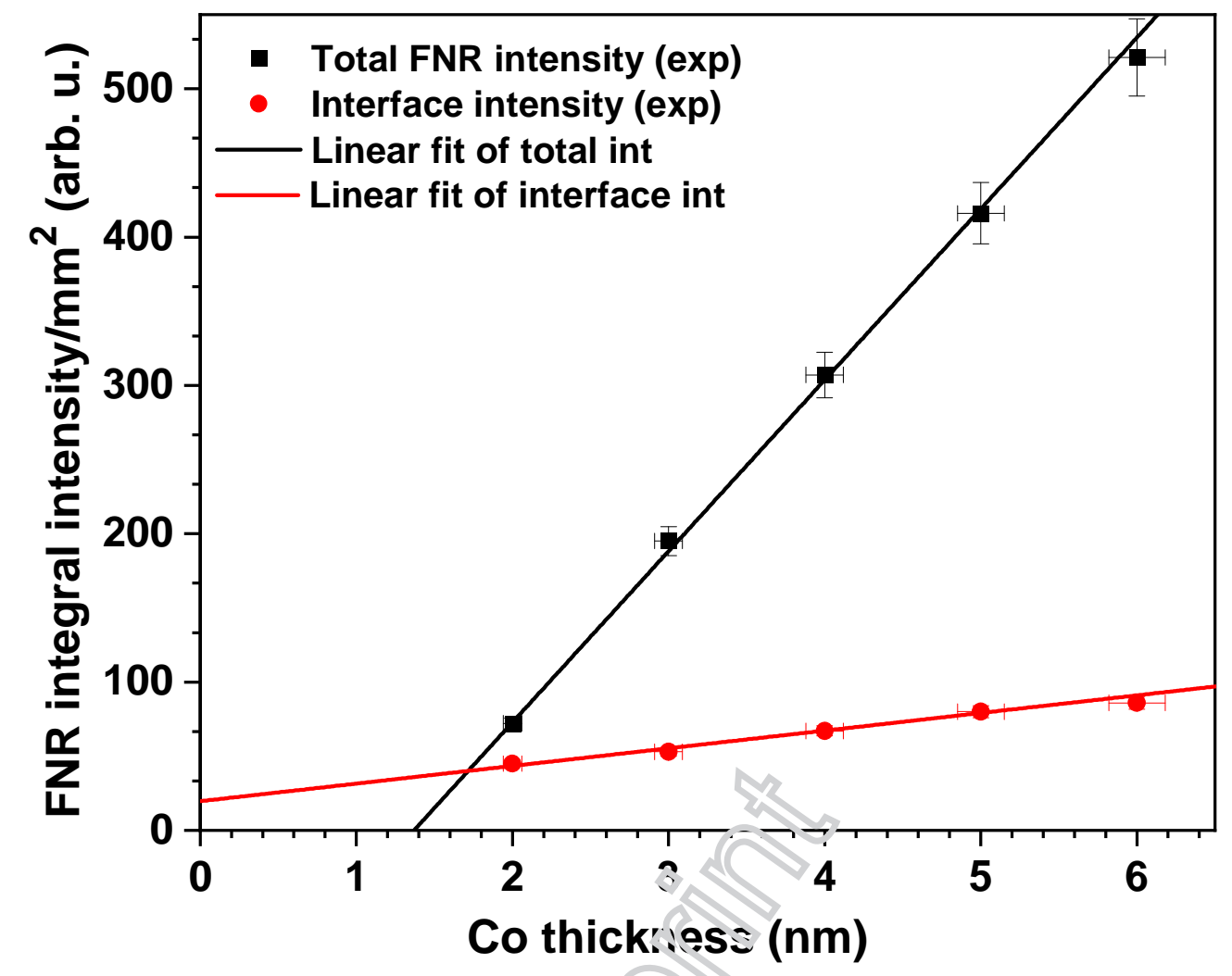

FIG. 2: Black symbols: total FNR intensities of the samples versus the deposited Co thickness. The linear fit (black line does not pass through the origin, evidencing Co dead layers. Red symbols: integral intensities of the part of the spectra below $200 \mathrm{MHz}$. The red line is the linear fit of the $<200 \mathrm{MHz}$ integral intensities.

To investigate the asymmetry of the Co interfaces, samples with specific architectures were prepared: $\mathrm{Cu} / \mathrm{Fe} / \mathrm{Co} \quad(6 \mathrm{~nm}) / \mathrm{ZnTPP} \quad(20 \mathrm{ML})$ and $\mathrm{Cu} / \mathrm{ZnTPP} \quad(20 \mathrm{ML}) / \mathrm{Co} \quad(6 \mathrm{~nm}) / \mathrm{Fe}$ heterostructures. Such architectures allow to split the contributions of the two buried Co interfaces in two distinct frequency ranges allowing the investigation of single buried interfaces. Indeed, when Co atoms have Fe atoms among their nearest neighbours, the Co resonance frequency increases [24-26] and thus the $\mathrm{Co} / \mathrm{Fe}$ interface contributions will appear at high frequency and the low frequency contributions will originate from the single Co/ZnTPP or ZnTPP/Co interfaces only. The FNR spectra of these two samples are shown in Figure 3. The comparison of the spectra of the top Co/ZnTPP and bottom ZnTPP/Co interfaces shows that below $140 \mathrm{MHz}$ the two interfaces result in identical and superimposable contributions, as observed for the ZnTPP/Co/ZnTPP sandwich samples. However, between $140 \mathrm{MHz}$ and 200 $\mathrm{MHz}$, the two spectra depart significantly. The FNR signal remains weak when the ZnTPP 
layer is deposited on top of Co while the FNR intensity increases dramatically when Co is deposited on top of the ZnTPP layer. It shows that a much larger amount of Co atoms is involved in the interface region when Co is deposited on top of ZnTPP than when ZnTPP is deposited on top of Co. The slope of the FNR integral intensities versus the deposited Co thickness, Figure 1, corresponds to the FNR experimental intensity resulting from the deposition of $1 \mathrm{~nm}$ of Co. Using this value, the amount of Co atoms involved in specific frequency ranges of the spectra can be estimated. From the integral intensity of the low frequency contributions $(<200 \mathrm{MHz})$ it is therefore possible to estimate the amount of Co atoms involved in the interfaces with ZnTPP. When ZnTPP is deposited on top of Co, the thickness of Co involved in the interface is of the order of $0.4 \mathrm{~nm}$. Considering that a perfectly flat interface would already involve $0.2 \mathrm{~nm}$ (one atomic plane) [7,8] of $\mathrm{Co}$, an interface contribution of about $0.4 \mathrm{~nm}$ is rather small and is characteristic of a sharp interface. On the contrary, when Co is deposited on top of ZnTPP, the quantitative analysis shows that the interface contribution is much larger: it is of the order of $0.7 \mathrm{~nm}$ of Co thickness. This can be understood by considering that the interlayer distance between the organic planes is much larger $(0.35 \mathrm{~nm}-0.4 \mathrm{~nm})$ than that of a metal, therefore a small roughness at the organic layer scale will directly impact a large number of Co atoms. It is thus not surprising to observe that the amount of Co atoms involved in the interface is much larger when Co is deposited on top of ZnTPP than when ZnTPP is deposited on top of Co. It ex lains why the top interface extends over a large thickness and why in the hybrid sandivich layers, a minimum of $5 \mathrm{~nm}$ of Co is required to complete the formation of the interfar ss. Sompared to other metal/organic systems, when ZnTPP is deposited on top of Co the interiac is rather sharp (for example it extends to about $2 \mathrm{~nm}$ when $\mathrm{C}_{60}$ is deposited on top of $:$ - 30 ]) while it is more extended when Co is deposited on top of ZnTPP.

Considering the shape of the interface FNIR spectra, none did show any well-defined, resolved, interfacial environment. Interface spectra consist in unstructured low frequency tails. This suggests that there are no strong interactions between the Co atoms and the organic layer (in other words no chemical ionic/covalent bonds). Indeed, in the case of Co atoms bonded to carbon atoms, well defined FNR lines have been reported [27]. In addition, considering previous works in inorganic films and multilayers, when interfaces are well defined sharp lines are observed (examples are given in [7] and [8]). This is mostly due to the fact that all Co atoms experience the same local environment at the interface. When interfaces are disordered more lines appear and broaden the FNR response. Transposing this in the case of the Co/organic systems if strong chemical bonds exist between Co and the organic molecules they should result in a well-defined local environment for the interfacial Co atoms and therefore in a welldefined resonance line. On the contrary in the case of weak van der Waals interactions the position of the molecules on top of the Co films will be easily influenced by surface defects. Consequently, there will be no specific relationship between the position of the Co surface atoms and the organic molecules. Therefore, the Co surface atoms will experience a large variety of local environments resulting in a large dispersion of their resonance frequencies. Since, even in the case of the sharpest interface (ZnTPP on top of Co) no well-defined interfacial line could be identified, this suggests that there is no well-defined relationship 
between the position of the surface Co atoms and the ZnTPP molecules. The most likely explanation is that the interfacial interactions are of weak van der Waals type.

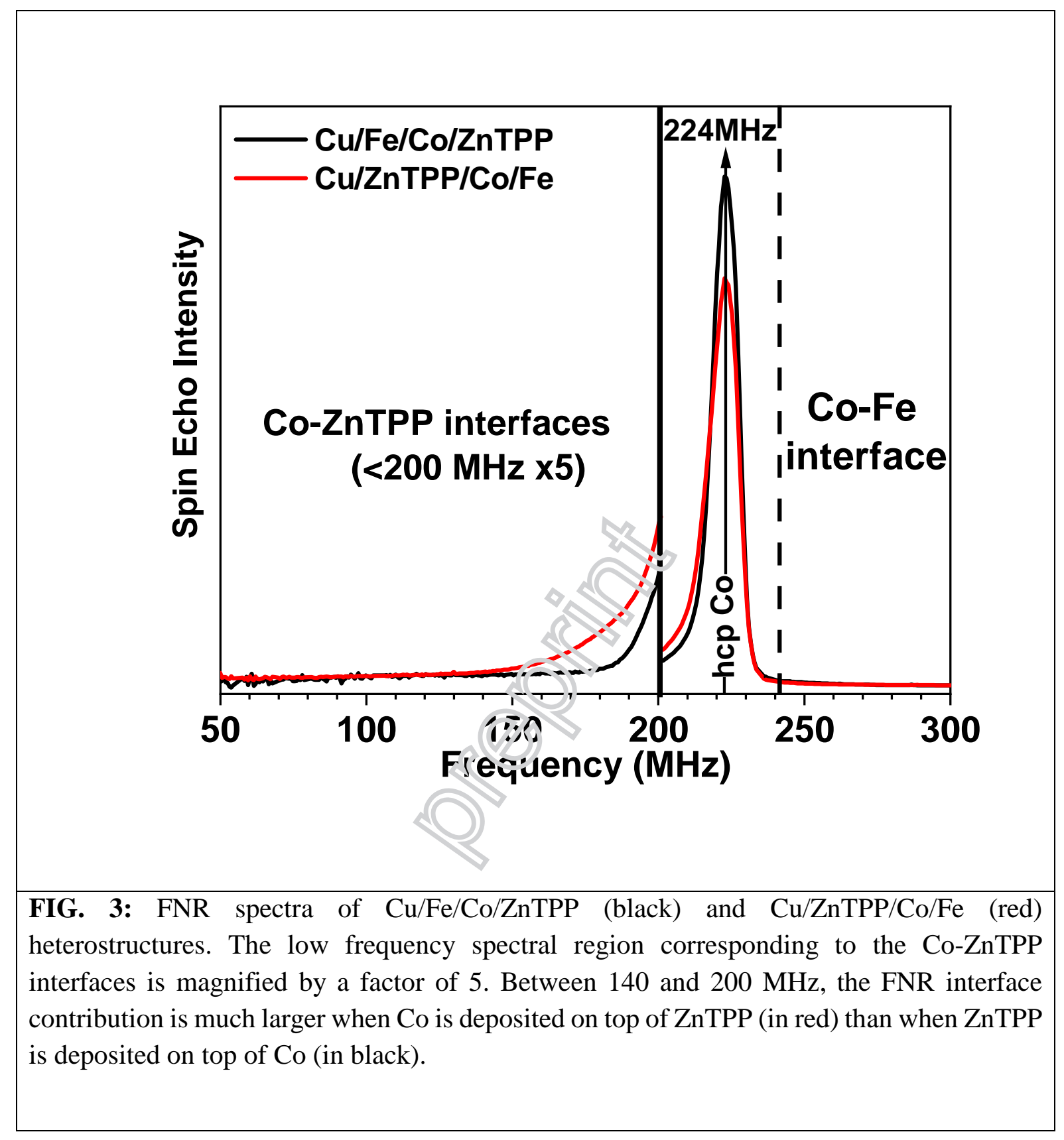

It has been reported in previous works that organic molecules can have a large impact on the surface anisotropy of the magnetic films. It can result both to interfacial hardening or interfacial softening [31]. This effect can also be investigated by FNR since in addition to the provided structural and chemical information, FNR also allows probing the local anisotropy of the samples. Indeed, as briefly explained in the methods part the procedure used to establish the FNR spectra [7,8] also provides the so-called site dependent restoring field. In the studied samples the local restoring field can be identified as the local magnetic anisotropy field. This 
information is seldom extracted from the FNR data, but recently it was successfully used to understand the origin of the increase in anisotropy of permanent magnets doped with Co [32]. This approach allows to probe the magnetic anisotropy field of the Co atoms at the interfaces with respect to the magnetic anisotropy of the bulk (the inside) of the Co layers. Figure 4 shows the frequency dependent restoring field obtained on both single Co/ZnTPP and ZnTPP/Co interfaces. The behaviour of the two samples is similar as the largest local magnetic anisotropy is obtained at $224 \mathrm{MHz}$ and corresponds to the Co atoms far from the interfaces, situated inside the Co layers. The low frequency $(<200 \mathrm{MHz})$ restoring field is assigned to the magnetic anisotropy field of the Co/ZnTPP and ZnTPP/Co interfaces and it is typically twice smaller than the anisotropy of the bulk Co atoms. Since the interfaces of the Co layers always show a smaller anisotropy field than the bulk part of the Co layers, it is thus possible to conclude that no interface hardening effect is observed in these layers. Indeed, if interface hardening would take place the interface anisotropy field should be larger than the anisotropy field of the bulk part of the Co layers, what is clearly not the case. It is possible that the hardening effect does exist at a local scale but is annihilated by the interfacial disorder. A similar effect was observed in metallic films and multilayers where the predicted increase of interface anisotropy was only observed for samples showing almost perfectly flat interfaces [8]. Since the Co/ZnTPP interface is much sharper than the ZnTPP/Co interfare, the effect of the interface disorder should result in significant differences in their bu!k is interface anisotropy field ratios. However, this is not observed experimentally sirac ior both samples this ratio is close to two. The only difference that is observed between th san ples is an overall shift of the anisotropy fields. This shows that the samples' average saisot opy is slightly different. The sample with the largest interface disorder has a smaller averacf anisotropy than the other one. Most likely, the absence of interface hardening is due $6, n=$ weak van der Waals interactions taking place at the Co/ZnTPP interfaces. These wean interactions combined with some interfacial disorder result in a reduction of the magnetic inisotropy at the interfaces. 


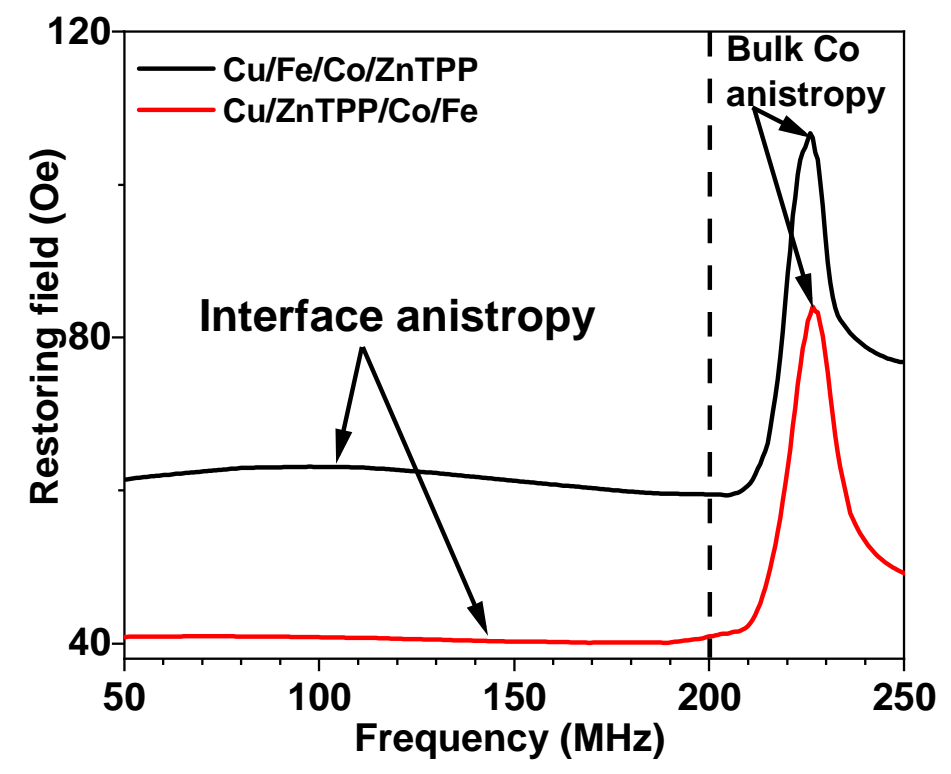

FIG. 4: Frequency dependent FNR restoring field. R toring field can be identified as the in plane local magnetic anisotropy field in the sample . Pis anisotropy of the interfaces $(<200$ $\mathrm{MHz}$ ) is much smaller than the anisotropy of the rail part of the Co films (>200 MHz).

\section{Conclusion}

Co layers sandwiched between ZnTPF ia. ers as well as single buried Co/ZnTPP and ZnTPP/Co interfaces have been studied in this work. No well-resolved FNR contribution could be attributed to Co atoms situated at the Co-ZnTPP interfaces. This suggests that no strong chemical bonds exist between the Co atoms and the organic layers at the interfaces and that the interfacial interactions are governed by weak van der Waals interactions. In addition, the analyses of the FNR spectra showed that the interfaces are strongly asymmetric. When ZnTPP is deposited on top of Co (Co/ZnTPP), it forms a rather sharp interface. On the contrary, when Co is deposited on top of ZnTPP (ZnTPP/Co) a much larger interfacial zone is formed and the deposition of up to $5 \mathrm{~nm}$ of $\mathrm{Co}$ is required to complete the interface formation. Finally, the interface magnetic anisotropy field was probed through the FNR restoring field and showed that the magnetic anisotropy field of the interfacial Co atoms is twice smaller than the magnetic anisotropy field of the Co atoms in centre of layers. This is attributed to the weak van der Waals interfacial interactions and/or to the interfacial disorder.

\section{Acknowledgements}

This work was funded by the French National Research Agency (ANR) through the Programme d'Investissement d'Avenir under contract ANR-11-LABX-0058_NIE within the 
Investissement d'Avenir program ANR-10-IDEX-0002-02. We acknowledge Christophe Kieber for technical assistance and the IdEx program of the University of Strasbourg for a scholarship for Garen Avedissian.

\section{References}

[1] V. Dediu, M. Murgia, F. C. Matacotta, C. Taliani, and S. Barbanera, Solid State Commun 122,181-184 (2002).

[2] P. P. Ruden and D. L. Smith, J. Appl. Phys. 95, 4898-4904 (2004).

[3] Z. H. Xiong, D. Wu, Z. V. Vardeny and J. Shi, Nature 427, 821-824 (2004).

[4] S. Majumdar, H. S. Majumdar, R. Laiho, and R. Osterbacka, J. Alloys Compd. 423, 169171 (2006).

[5] T. Moorsom et al., Phys. Rev. B 90, 125311 (2014).

[6] S. Sanvito, Nat. Phys. 6, 562-564 (2010).

[7] P. Panissod and C. Meny, Appl. Magn. Reson 14, 447-460 (2000).

[8] P. Panissod, C. Meny, M. Wojcik and E. I cur ka, Mater. Res. Soc. Symp. Proc. 475, 157168 (1997).

[9] J. L. Bubendorff, E. Beaurepaire, C. Meıy and J. P. Bucher, J. Appl. Phys. 83, 7043-7045 (1998).

[10] M. Vélez, C. Meny, S. M. Valvidaies, J. Diaz, R. Morales, L. M. Alvarez-Prado, P. Panissdo and J. M, Alameda, Eur. Phys. J. B 41, 517-524 (2004).

[11] A. Azizi, A. Sahari, M. L. Felloussia, G. Schmerber, C. Meny and A. Dinia, Appl. Surf. Sci. 228, 320-325 (2004).

[12] M. Wójcik, J. P. Jay, P. Panissod, E. Jedryka, J. Dekoster and G. Langouche, Zeitschrift für Physik B 103, 5-12 (1997).

[13] H. El Fanity, K. Rahmouni, M. Bouanani, A. Dinia, G. Schmerber, C. Meny, P. Panissod, A. Cziraki, F. Cherkaoui and A. Berrada, Thin Solid Films 318, 227-230 (1998).

[14] J. Dekoster, E. Jedryka, C. Meny and G. Langouche, J. Magn. Magn. Mater. 121, 69-72 (1993).

[15] K. Le Guen, M. -H. Hu, J. -M. Andre, S. K. Zhou, H. Ch. Li, J. T. Zhu, Z. S. Wang, C. Meny, A. Galtayries and P. Jonnard, Appl. Phys. Let. 98, 251909 (2011). 
[16] E. Jedryka, M. Wójcik, S. Nadolski, D. Kubinski, M. Parsons and H. Holloway, J. Appl. Phys. 91, 7191-7193 (2002).

[17] M. Wójcik, C. Christides, E. Jedryka, S. Nadolski and I. Panagiotopoulos, Phys. Rev. B 63, 012102 (2001).

[18] C. Meny, P. Panissod, P. Humbert, J. P. Nozieres, V. S. Speriosu, B. A. Gurney and R. Zehringer, J. Magn. Magn. Mater. 121, 406-408 (1993).

[19] Y. F. Liu, J. Luo, Y. Shin, S. Moldovan, O. Ersen, A. Hebraud, G. Schlatter, C. PhamHuu and C. Meny, Nat. Commun. 7, 11532 doi: 10.1038/ncomms 11532 (2016).

[20] A. S. Andreev, O. B. Lapina, J. B. d'Espinose de Lacaillerie and A. A. Khassin, J. Struct Chem, 54, S102-S110 (2013).

[21] M. Malinowska, M. Wójcik, S. Nadolski, E. Jedryka, C. Meny, P. Panissod, M. Knobell, A. D. C. Viegas and J. E. Schmidt, J. Magn. Magn. Mater. 198-199, 599-601 (1999).

[22] R. Speight, A. Wong, P. Ellis, P. T. Bishop, T. I. Hyde, T. J. Bastow and M. E. Smith, Phys. Rev. B 79, 054102 (2009).

[23] V. Scarani, H. De Riedmatten and J. P. Anserm f, , 1 ppl. Phys. Lett. 76, 903 (2000).

[24] G. Avedissian, J. Arabski, J. A. Wytko, Weiss, and C. Meny, Adv. Funct. Mater. doi:10.1002/adfm.202005605 (2020).

[25] M. Malinowska, C. Meny, E. Jedry d and Panissod, J. Phys.: Condens. Matter. 10, 4919-4928 (1998).

[26] C. Meny, E. Jedryka and P. Par's sor. J. Phys.: Condens. Matter. 5, 1547-1556 (1993).

[27] E. Dujardin, C. Meny, P. Panissod, J. P. Kintzinger, N. Yao and T. W. Ebbesen, Solid State Comm. 114, 543-546 (2000).

[28] S. Entani, M. Kiguchi, S. Ikeda and K. Saiki, Thin Solid Films 493, 221-225 (2005).

[29] H. Fritzsche, J. Kohlhepp, and U. Gradmann, Phys. Rev. B 51, 15933-15942 (1995).

[30] S. Mallik, A. Syed Mohd, A Koutsioubas, S. Mattauch, B. Satpati, T. Brückel and S. Bedantam, Nanotechnology 30, 435705 (2019).

[31] K. Bairagi et al. Phys. Rev. Lett. 114, 247203 (2015).

[32] H. Nakamura, T. Waki, Y. Tabata and C. Meny, J. Phys.: Mater. 2, 015007 (2019). 